\title{
Questionário de Fusão Cognitiva (CFQ): novas evidências de validade e invariância transcultural
}

\author{
Evandro Morais Peixoto ${ }^{1}$ \\ Giovanna Corte Honda $\mathbb{1}^{2}$ \\ Joel Gagnon $\mathbb{1}^{3}$ \\ Glaucia M. Ataka da Rocha $\mathbb{( i D}^{4}$ \\ Tatiana de Cássia Nakano ${ }^{5}$ \\ Daniela Sacramento Zanini ${ }^{6}$ \\ Marcos Alencar Abaide Balbinotti ${ }^{3}$ \\ ${ }^{1}$ Universidade de Pernambuco, PE, Brasil. \\ ${ }^{2}$ Universidade Nove de Julho (UNINOVE), SP, Brasil. \\ ${ }^{3}$ Université du Québec à Trois-Rivieres (UQTR), Quebec, Canadá \\ ${ }^{4}$ Universidade Federal do Tocantins (UFT), TO, Brasil. \\ ${ }^{5}$ Pontificia Universidade Católica de Campinas (PUCC), SP, Brasil. \\ ${ }^{6}$ Pontificia Universidade Católica de Goiás (PUCG), GO, Brasil.
}

\section{Resumo}

A fusão cognitiva é um conceito chave na Terapia de Aceitação e Compromisso, mecanismo em que a pessoa se funde com seus pensamentos e os toma como se fossem fatos reais. Os objetivos desta pesquisa foram de estimar novas evidências de validade do Questionário de Fusão Cognitiva (CFQ) e de testar a invariância dos itens entre amostra brasileira e franco-canadense. Participaram desta pesquisa 578 adultos brasileiros e 676 adultos franco-canadenses. A Análise Fatorial Exploratória revelou adequação da estrutura unidimensional, conforme hipótese teórica. O Rating Scale Model demostrou índices de dificuldade entre -0,42 e 0,69 e índices ajustes adequados (Infit/Outfit) entre 0,79 e 1,45 para os itens de ambas as versões e descrição sumarizada dos níveis de theta dos participantes. A Análise de DIF apontou dois itens que não suportavam invariância no parâmetro dificuldade em função das diferenças culturais. Contudo, observou-se invariância destes parâmetros quando avaliados em função do gênero dos participantes de cada amostra.

Palavras chave: terapia de aceitação e compromisso-ACT, validade, psicoterapia, teoria de resposta ao item-IRT, psicologia intercultural.

\section{Cognitive Fusion Questionnaire (CFQ): new validity evidences and cross-cultural invariance}

\begin{abstract}
The cognitive fusion is a key concept in Acceptance and Commitment Therapy-ACT. It is a mechanism in which the person merges with his/hers thoughts as if they were real. The objectives of this study were to estimate new validity evidences of the Brazilian version of the Cognitive Fusion Questionnaire (CFQ) and to test the items invariance among Brazilian and French-Canadian sample. The sample comprised 578 Brazilian adults and 676 French-Canadian adults. The Exploratory Factor Analysis showed adjustment of the one-dimensional structure, confirming the theoretical hypothesis. The Rating Scale Model showed difficulty indexes between -0, 42 e 0, 69, god fit indexes (Infit/Outfit) between 0,79 e 1,45 to both items versions, as well as summarized description of participants' theta levels. The DIF analysis showed that two items violated the invariance of the difficulty parameter due to cultural clashes. However, it was observed invariance of these parameters when the participants'gender was considered in the analysis.
\end{abstract}

Keywords: acceptance and commitment therapy-ACT, validity, psychotherapy, Item Response Theory-IRT, cross-cultural psychology.

\section{Cuestionario de Fusión Cognitiva (CFQ): nuevas evidéncias de Validez e invarianza transcultural}

\section{Resumen}

La fusión cognitiva es un concepto clave en la Terapia de Aceptación y Compromiso, mecanismo en que la persona se funde con sus pensamientos, como si estos son reales. Los objectivos de esta investigación fueran evaluar nuevas evidéncias de validez de la versión brasileña del Cuestionario de Fusión Cognitiva (CFQ) y testar la invarianza de los ítems entre muestra brasileña y francocanadiense. Análisis Factorial exploratorio reveló adecuación de la estructura unidimensional, según la hipótesis teórica. El Rating Scale Model mostró índice de dificultad entre -0,42 e 0,69, índices de ajustes adecuados (Infit/Outfit), entre 0,79 e 1,45, para los ítems de las dos versiones, así como análisis descritivo de los niveles theta de los participantes. Análisis del DIF mostró dos ítems que no suportavan invarianza del parámetro dificultad en función de las diferencias culturales. Sin embargo, se observó invarianza de estos parámetros cuando evaluados en función de lo género de los participantes de cada muestra.

Palabras clave: terapia de aceptación y compromiso-ACT, validad; psicoterapia, teoria de respuesta al ítem-IRT, psicologia intercultural. 
A Terapia de Aceitação e Compromisso (Acceptance and Commitement Therapy-ACT) é uma modalidade de terapia cognitivo-comportamental que explica o funcionamento humano por meio da flexibilidade psicológica dos indivíduos. É entendida como a capacidade do sujeito em sentir integralmente o momento atual e manter ou modificar seu comportamento, dependendo dos valores escolhidos por ele (Barbosa \& Murta, 2014; Gillanders et al., 2014; Haeys, Luoma, Bond, Masuda, \& Lillis, 2006).

Quando a pessoa vivencia uma situação difícil, seus valores podem ser colocados em segundo plano, promovendo inflexibilidade psicológica. Nela, as ações são guiadas pela linguagem e são pouco motivadas pelos valores, o que pode gerar um comportamento disfuncional (Barbosa \& Murta, 2014). Neste caso, o indivíduo não consegue agir de maneira flexível no que tange a pensamentos, emoções e sensações, avaliadas como negativas.

Silva (2015) aponta que a inflexibilidade psicológica é causa de sofrimento humano e de diferentes tipos de psicopatologias como, por exemplo, a compulsão alimentar. Nesse sentido, a ACT apresenta vasta sustentação empírica acerca de seus resultados e é considerada bem-sucedida em tratamentos para dor crônica, depressão, abuso de substâncias e ansiedade (Barbosa \& Murta, 2014; Dionne et al., 2016).

O objetivo do terapeuta que trabalha com essa abordagem é o de auxiliar o cliente a se tornar mais consciente no que diz respeito aos objetivos de sua própria vida e aos valores que guiam sua conduta, auxiliando-o a lidar com as circunstâncias de mudanças. Neste sentido, busca-se a flexibilidade psicológica por meio de seis processos centrais na $\mathrm{ACT}$, que são: atenção presente, valores, compromisso, self como contexto, aceitação e defusão (Gillanders et al., 2014; Haeys et al., 2006; Hayes, Pistorello \& Biglan, 2008). De acordo com Barbosa e Murta (2014) e Haeys et al. (2006), cada um desses processos possui um polo inverso, que promove a inflexibilidade psicológica, como por exemplo: atenção rígida ao passado e/ou ao futuro, objetivos e valores pouco claros, dúvidas e estratégias evasivas, esquiva e fusão cognitiva.

A ideia de fusão cognitiva é uma peça chave na ACT. Ela é entendida como um mecanismo em que a pessoa se funde com seus pensamentos e os toma como se fossem fatos reais (Ruiz, Suárez-Falcón, Riaño-Hernandez, \& Gillanders, 2017; Silva, 2015). Quando se encontra emaranhado em seus próprios pensamentos, o indivíduo não consegue reconhecer que seu comportamento é regulado por conteúdo cognitivo e não pela realidade, portanto, acaba ignorando totalmente o contexto ambiental e sua própria experiência (Barbosa \& Murta, 2014; Dionne et al., 2016).

Nesse estado de fusão, se o conteúdo privado é avaliado como negativo, ocasionando repulsa, angústia, dor, entre outros, o indivíduo tende a desenvolver estratégias para evitar sentir tais experiências, com intuito de minimizar o incômodo acarretado por elas (Ruiz et al., 2017). Silva (2015) aponta que o mecanismo de fusão cognitiva está associado a menor qualidade de vida, pois a esquiva, nesse caso, pode contribuir para a inflexibilidade psicológica e favorecer o desenvolvimento de psicopatologias. Além disso, a fusão cognitiva pode reforçar a rigidez comportamental, causar sofrimento e limitar as opções e experiência da pessoa, pois existe a tendência a se esquivar de situações que provocam sensações desagradáveis (Barbosa \& Murta, 2014), o que, por sua vez, tende a reforçar ainda mais a fusão cognitiva. A ACT propõe perfazer o processo inverso, conhecido como defusão cognitiva.

A defusão é uma técnica que visa modificar o modo como a pessoa se conecta com a própria experiência mental, diminuindo o poder dos eventos privados sobre o comportamento do sujeito (Barbosa \& Murta, 2014; Gillanders et al., 2014. Haeys et al., 2006). O intuito é atenuar a tendência de tratar os pensamentos como se fossem fatos reais. Desta forma, possibilita a flexibilização dos processos cognitivos e o aumento do contato com a experiência vivida (Haeys et al., 2006; Ruiz et al., 2017).

A maneira como a pessoa se relaciona com os próprios pensamentos pode explicar como ocorre o funcionamento de seu comportamento, bem como o entendimento de possíveis psicopatologias. Por isso, compreender de forma mais objetiva a fusão cognitiva, pode ser muito vantajoso para o psicólogo. Neste sentido, Gillanders et al. (2014) desenvolveram o Questionário de Fusão Cognitiva (CFQ - Cognitive Fusion Questionnaire), uma medida de autorrelato composta por sete itens, dispostos em uma escala Likert de 7 pontos ( $1=$ nunca a $7=$ sempre). $O$ respondente deve analisar o conteúdo dos itens e, a partir deles, assinalar em que grau fica fusionado com seus pensamentos. Os escores podem variar entre 7 e 49, sendo que, quanto maior a pontuação, maior o grau de fusão cognitiva.

A avaliação das propriedades psicométricas do questionário original foi realizada com 1800 pessoas, divididas em sete amostras diferentes. Observou-se que o instrumento apresentava boas evidências de validade e precisão: estrutura interna unifatorial, consistência interna avaliada por meio do coeficiente alfa de Cronbach entre 0,88 a 0,92 e estabilidade temporal medida por meio de teste-reteste, com intervalo de 
quatro semanas $(r=0,80)$. Além disso, o questionário demostrou boas evidências de validade com base na relação com outras variáveis, correlacionando-se de acordo com a perspectiva teórica com medidas de diferentes construtos como aceitação, mindfulnness, ansiedade, depressão e satisfação com vida (Gillanders et al., 2014).

Devido às suas qualidades psicométricas e praticidade na aplicação, o CFQ foi traduzido e adaptado para diferentes países, mostrando resultados similares ao da escala original em todos eles. $\mathrm{Na}$ versão espanhola, Romero-Moreno, Márques-González, Losada, Gillanders e Fernández-Fernández (2014), por meio da Análise Fatorial Confirmatória (AFC), verificaram a adequação da estrutura unifatorial e bom índice de consistência interna $(\alpha=0,87)$ frente a uma amostra de cuidadores de pacientes com demência. Também apresentaram associações significativas na direção esperada teoricamente entre o CFQ e variáveis de coping, satisfação com vida, ruminação, depressão e ansiedade.

Na versão colombiana, Ruiz et al. (2017) observaram, por meio da $\mathrm{AFC}$, a adequação da estrutura unifatorial para três diferentes amostras: universitários, população geral (que respondeu ao instrumento online) e amostra clínica (pacientes em processos psicoterápicos), bem como bons índices de consistência interna $(0,89 ; 0,93$; e 0,93 , respectivamente). Os autores também observaram a invariância do modelo de medida entre as diferentes amostras, além de associações significativas entre CFQ e medidas de aceitação, satisfação com a vida, mindfulness, depressão, ansiedade e estresse.

Na versão franco-canadense, Dionne et al. (2016), por meio da Análise Fatorial Exploratória (AFE), obtiveram resultados semelhantes em três diferentes amostras: universitários (que responderam a versão online), universitários (que usaram a versão em lápis e papel) e pacientes com dor crônica. Os resultados apontaram para solução unifatorial e coeficientes alfa de Cronbach iguais a 0,94, 0,93 e 0,94, respectivamente, e níveis de associação significativos com outras variáveis como aceitação, ansiedade, depressão, inflexibilidade psicológica, dor e mindful attention. Outras versões do instrumento apresentaram propriedades psicométricas similares à proposta original, tais como a chinesa (Weichen, Yang, Li, Hui-Na, \& Zhuo-Hong, 2014), catalã (Solé et al., 2015) e italiana (Dell'Orco, Prevedini, Oppo, Presti, \& Moderato, 2012).

No Brasil, estudos de tradução e adaptação do CFQ foram realizados por Lucena-Santos, Carvalho, PintoGouveia, Gillanders e Oliveira (2017). Na ocasião, os autores também avaliaram diferentes propriedades psicométricas do instrumento, tais como estrutura interna, precisão e invariância do instrumento frente a três amostras compostas por mulheres: população geral, estudantes universitárias e pacientes em tratamento para obesidade e sobrepeso. Resultados obtidos por meio da AFC indicaram índices de ajuste considerados muito bons para o modelo unifatorial, bem como indicadores de forte invariância do instrumento frente às diferentes amostras avaliadas. Lucena-Santos et al. (2017) também verificaram bons indicadores de precisão do CFQ (alfas entre 0,92 e 0,94 ), em diferentes amostras. Além disso, evidenciaram associações positivas significativas entre fusão cognitiva e ruminação, depressão, ansiedade, estresse e inflexibilidade psicológica e associações negativas significativas com mindfulness e decentering.

Os estudos descritos demonstram que o CFQ revela possibilidades abrangentes de uso, como em avaliação pré ou pós-intervenção, no intuito de caracterizar um possível progresso do cliente (Gillanders et al., 2014) ou para compor o instrumental, disponível aos pesquisadores, que tem por objetivo verificar como a fusão cognitiva relaciona-se com outras variáveis de interesse (Dionne et al., 2016), haja vista que esse construto se mostrou associado a diferentes distúrbios psicológicos e influências comportamentais em diferentes culturas (Lucena-Santos et al., 2017; Ruiz et al., 2017).

Embora o CFQ tenha se mostrado apropriado a diferentes línguas e culturas, como o português brasileiro, o instrumento ainda não conta com evidências de validade para a população masculina, no Brasil. Além disso, as pesquisas realizadas com o CFQ basearam-se na Teoria Clássica dos Testes (TCT). Neste sentido, a utilização da Teoria de Resposta ao Item (TRI) pode ser vista como um modelo psicométrico complementar que possibilita novas respostas quanto ao desenvolvimento e à validação do CFQ, possibilitando melhor compreensão quanto às forças e fragilidades dos itens que compõem esse instrumento.

Outra importante lacuna referente ao CFQ diz respeito à falta de estudos interculturais que comparem a invariância dos parâmetros do questionário entre amostras oriundas de diferentes países. Conforme destacado pela International Test Commission (2016), as evidências de invariância dos parâmetros de um instrumento devem ser consideradas um pré-requisito para a comparação de populações por meio dos escores. A fim de oferecer contribuições para o preenchimento destas lacunas, a presente pesquisa teve como objetivos estimar novas evidências de validade do CFQ frente à população brasileira e testar a invariância dos parâmetros dos itens entre amostra brasileira e francocanadense por meio da TRI. 


\section{Método}

\section{Participantes}

Duas amostras diferentes foram acessadas. A primeira, composta por 578 participantes brasileiros, representantes de diferentes regiões do País sendo $70,3 \%$ do Sudeste, $19,6 \%$ do Sul; $6,8 \%$ do CentroOeste, $2,6 \%$ do Nordeste, e $0,7 \%$ do Norte, com idades que variaram entre 15 e 86 anos $(M=36,54 \pm 12,74)$, dos quais 76,8\% eram mulheres. Quanto ao nível de escolaridade $91,1 \%$ possuíam nível superior completo e $8,9 \%$ ensino médio completo. A segunda amostra, foi composta por 676 estudantes universitários canadenses, provenientes da província do Québec, com idades que variaram entre 18 e 60 anos $(\mathrm{M}=23,89 \pm 5,43)$ dos quais $78,1 \%$ eram mulheres. Quanto ao nível de escolaridade, $75 \%$ possuíam nível superior incompleto e $25 \%$ nível superior completo. O critério para escolha das amostras se deu pelo fato de os participantes de ambas as nacionalidades terem respondido à versão online do instrumento.

\section{Instrumentos}

- Questionário de Fusão Cognitiva (QFC) (Gillanders et al., 2014), tem por objetivo avaliar o grau em que as pessoas tendem a se fundir com os pensamentos. É composto por sete itens respondidos em uma escala Likert de 7 pontos ("nunca verdadeira" a "sempre verdadeira"). O respondente assinala a alternativa que melhor representa o conteúdo que o descreve. De acordo com estudo original, estes itens são dispostos em uma solução unifatorial com bons indicadores de precisão, com alfa de Cronbach entre 0,92 e 0,94 (Lucena-Santos, 2017).

- Escala de Satisfação com a Vida (ESV) (Diener, Emmons, Larsen, \& Griffin, 1985), visa avaliar a satisfação dos respondentes com as suas condições de vida atual em relação ao padrão de vida estabelecido por ele como desejável. A escala é composta por cinco itens respondidos em escala Likert de 7 pontos que variam entre "discordo plenamente" a "concordo plenamente". No Brasil, estudos referentes à estrutura interna da ESV têm corroborado a proposta original de solução unifatorial com bons indicadores de precisão, com alfa de Cronbach entre 0,72 e 0,80 (Gonçalves \& Kapczinski, 2008; Gouveia et al., 2003).

- Questionário Sociodemográfico, ferramenta que proporciona a investigação das principais características da amostra como idade, sexo e nível de escolaridade.

\section{Procedimentos, considerações éticas e coleta de dados}

A pesquisa foi realizada após aprovação do projeto de pesquisa pelo Comitê de Ética e Pesquisa com Seres Humanos sob o número de protocolo (CAAE 0118.0.272.000-11). Em ambas as amostras os participantes foram recrutados por meio de anúncios em mídias sociais (por exemplo, Facebook) convidando-os a participar de uma pesquisa online através do software Google questionário. Para tanto, foi solicitado aos participantes que lessem o Termo de Consentimento Livre e Esclarecido e, após concordância em participar, para assinarem o documento. Foi indicado também que, para responder à pesquisa, era necessário possuir idade igual ou superior a 18 anos. Especificamente para amostra canadense, os participantes foram informados da possível recompensa pela participação (seis cartõespresente MASTERCARD ${ }^{\circ}$ no valor de $25 \$$ que seriam sorteados).

\section{Análises estatísticas}

De acordo com os objetivos da pesquisa, diferentes procedimentos foram empregados. Para a avaliação da estrutura interna da versão brasileira do CFQ recorreuse à Analise Fatorial Exploratória (AFE) com método de estimação Unweightes Lesast Squares (ULS) e rotação oblíqua Promax, tendo como base uma matriz de correlações policóricas (Holgado-Tello, ChacónMoscoso, Barbero-García, \& Vila-Abad, 2010). Para estimação do número de fatores a serem extraídos, diferentes critérios foram avaliados, tais como: Análise Paralela (AP) baseada nos Minimum Rank Factor Analysis (Timmerman \& Lorenzo-Seva, 2011); método Hull (Lorenzo-Seva, Timmerman, \& Kiers, 2011); cargas fatoriais superiores a 0,30 e interpretabilidade da solução fatorial (Costello \& Osborne, 2005). Avaliouse a consistência interna por meio da versão ordinal do coeficiente alfa de Cronbach, tendo como referência valores superiores a 0,70. Estas análises foram realizadas com apoio do software estatístico Factor 10.3 (Lorenzo-Seva \& Ferrando, 2006). Estimaramse evidências de validade divergentes por meio da associação com a variável satisfação com a vida, por meio da estimativa do coeficiente de correlação de Pearson. Espera-se, teoricamente, que o nível de fusão cognitiva apresentado pelo sujeito demonstre relação inversa moderada com satisfação com a vida (Gillanders et al., 2014; Romero-Moreno et al., 2014; Ruiz et al., 2017).

Para estimação dos parâmetros dos itens empregou-se Rating Scale Model com método de estimação Joint maximum likelihood. Este método permite 
verificar o nível de intensidade no construto dos participantes (theta), assim como os índices de dificuldade e índices de ajuste dos itens (infit, outfit), que se referem ao sumário dos resíduos entre respostas esperadas pelo modelo e as respostas observadas empiricamente. A estatística infit indica o nível de ajustamento aos padrões de resposta, quando valores de dificuldade dos itens estão próximos aos valores de theta da pessoa. A estatística outfit indica o nível de ajustamento aos padrões de respostas quando a diferença entre a dificuldade do item e o theta da pessoa é grande. Estes valores podem variar de 0 ao infinito, sendo que, valores próximos de 1 indicam bom ajuste e entre 0,50 e 1,50 são considerados adequados. Todas as análises concernentes à TRI foram realizadas com o software estatístico WINSTEPS (Linacre, 2015). Por fim, para estimar a invariância dos parâmetros dos itens em função da nacionalidade e gênero dos participantes recorreu-se a Differential Item Functioning (DIF). Esse procedimento descreve as diferenças na probabilidade de endosso ao item entre pessoas com o mesmo nível de traço latente. Foram adotados, como critérios para presença de DIF, valores de contraste superiores a 0,05 associados à probabilidade Mantel-Haenzel significativa (menor que 0,05$)$ (Linacre, 2015).

\section{Resultados}

A primeira etapa consistiu em avaliar os indicadores de adequação da matriz de correlação, os quais apresentaram resultados muito bons, $\mathrm{KMO}=0,907$, $\chi^{2}$ de Bartlett $=(21) 2112,9, p<0,001$ e que, portanto, davam suporte a fatorabilidade do conjunto de itens. Estes dados foram submetidos à AP e Método Hull para estimação do número de fatores a serem extraídos, sendo que ambos os métodos indicaram a pertinência de extração de uma solução unifatorial (ver Tabela 1).

Conforme apresentado na Tabela 1, na AP apenas o primeiro fator apresentou porcentagem de variância explicada superior às variâncias explicadas estimadas a partir das matrizes geradas aleatoriamente (500 matrizes). Por meio do método Hull observou-se também que a solução unifatorial foi a que apresentou melhor equilíbrio entre índices de ajuste e graus de liberdade sendo, a solução fatorial sugerida pelas análises, capaz de explicar $70,70 \%$ da variância total dos itens. Observa-se que os itens apresentaram cargas fatoriais que variavam entre 0,66 e 0,81 , bem como comunalidades que variavam entre 0,44 e 0,60 demostrando serem bons representantes do construto medido pelo fator. Em relação à consistência interna observou-se coeficiente alfa de Cronbach igual a 0,89 , o que pode ser interpretado com bom indicador de precisão da solução fatorial. No que diz respeito às evidências de validade divergente, foram observados índices de correlação $r=-0,34$. Tais resultados indicaram que, quanto maiores os níveis fusão cognitiva apresentados, menores são os níveis de satisfação com a vida.

Uma vez obtidas as evidências de validade e precisão da versão brasileira do CFQ, verificou-se os parâmetros dos itens e características dos participantes por meio do Rating Scale Model. Os resultados são apresentados na Tabela 2, onde se observa os índices de dificuldade dos itens (b), índices de ajustes (Infit) Outfit), correlação entre escore dos itens e nível de Theta dos indivíduos, assim como a análise de DIF. Ressalta-se que tais estatísticas foram avaliadas para ambas as amostras, separadamente.

TABELA 1

Resultados da Análise Paralela, Método Hull e Análise Fatorial Exploratória para o CFQ

\begin{tabular}{|c|c|c|c|c|c|c|c|c|}
\hline \multirow{2}{*}{$\begin{array}{l}\text { Fator/ } \\
\text { Itens }\end{array}$} & \multicolumn{3}{|c|}{ Análise Paralela } & \multicolumn{3}{|c|}{ Método Hull } & \multicolumn{2}{|c|}{ Análise Fatorial } \\
\hline & $\begin{array}{c}\text { \% de variância } \\
\text { dados reais }\end{array}$ & $\begin{array}{l}\text { \% de variancia media } \\
\text { dados aleatórios }\end{array}$ & $\begin{array}{l}95 \% \text { dados } \\
\text { aleatórios }\end{array}$ & $C F I$ & $g l$ & $\begin{array}{c}\text { Valores } \\
\text { Scree test }\end{array}$ & $\begin{array}{c}\text { Carga } \\
\text { fatorial }\end{array}$ & $h^{2}$ \\
\hline 0 & & & & 0,00 & 7 & 0.000 & & \\
\hline 1 & $70,70^{*}$ & 29,10 & 37,50 & 0,94 & 14 & $15,14^{*}$ & 0,66 & 0,66 \\
\hline 2 & 11,00 & 23,70 & 28,80 & 0,99 & 29 & 0.00 & 0,77 & 0,77 \\
\hline 3 & 8,10 & 18,80 & 22,90 & & & & 0,67 & 0,67 \\
\hline 4 & 6,40 & 14,10 & 17,90 & & & & 0,74 & 0,74 \\
\hline 5 & 3,20 & 9,60 & 14,40 & & & & 0,72 & 0,72 \\
\hline 6 & 0,60 & 4,70 & 9,30 & & & & 0,71 & 0,71 \\
\hline 7 & 0,00 & 0,00 & 0,00 & & & & 0,81 & 0,81 \\
\hline$\alpha$ & & & & & & & 0,89 & \\
\hline
\end{tabular}

$\%=$ porcentagem; $95 \%=$ percentil $95 ; \mathrm{GFI}=$ Goodness of fit Index $; \mathrm{gl}=$ grau de liberdade; $\mathrm{h}^{2}=$ comunalidades. * numero de fatores a serem retidos conforme método hull. 
TABELA 2

Propriedades dos itens do CFQ e características dos participantes em função das diferentes amostras/países

\begin{tabular}{|c|c|c|c|c|c|c|c|c|c|c|}
\hline \multirow{3}{*}{ Item } & \multicolumn{10}{|c|}{ Parâmetros dos Itens } \\
\hline & \multicolumn{4}{|c|}{ Amostra brasileira } & \multicolumn{4}{|c|}{ Amostra franco-canadense } & \multirow{2}{*}{$\begin{array}{c}\text { DIF- } \\
\text { Contraste }\end{array}$} & \multirow{2}{*}{$\begin{array}{r}p \text { Mantel } \\
\text { Haenzel }\end{array}$} \\
\hline & $\theta$ & Infit & Outfit & $r$ Item- $\theta$ & $\theta$ & Infit & Outfit & $r$ Item- $\theta$ & & \\
\hline i1 & $-0,36$ & 1,09 & 1,11 & 0,72 & 0,31 & 1,15 & 1,19 & 0,80 & $-0,67$ & 0,00 \\
\hline i2 & 0,49 & 0,85 & 0,84 & 0,78 & 0,69 & 1,06 & 1,06 & 0,80 & $-0,19$ & 0,00 \\
\hline i3 & 0,43 & 1,18 & 1,16 & 0,72 & $-0,39$ & 1,44 & 1,45 & 0,79 & $\mathbf{0 , 8 3}$ & 0,00 \\
\hline i4 & 0,01 & 0,98 & 1,01 & 0,76 & 0,14 & 0,81 & 0,79 & 0,85 & $-0,13$ & 0,08 \\
\hline i5 & $-0,21$ & 1,02 & 0,99 & 0,76 & $-0,27$ & 0,79 & 0,79 & 0,86 & 0,06 & 0,34 \\
\hline i6 & $-0,54$ & 1,03 & 1,01 & 0,76 & $-0,42$ & 0,88 & 0,85 & 0,86 & $-0,12$ & 0,11 \\
\hline i7 & 0,11 & 0,83 & 0,80 & 0,79 & 0,03 & 0,81 & 0,83 & 0,85 & 0,08 & 0,35 \\
\hline $\bar{x}$ & 0 & 1,00 & 0,99 & & 0 & 0,99 & 1,00 & & & \\
\hline \pm & 0,36 & 0,12 & 0,12 & & 0,38 & 0,22 & 0,23 & & & \\
\hline Máx. & 0,49 & 1,18 & 1,16 & & 0,69 & 1,44 & 1,45 & & & \\
\hline \multirow[t]{2}{*}{ Mín. } & $-0,54$ & 0,83 & 0,80 & & $-0,42$ & 0,79 & 0,79 & & & \\
\hline & \multicolumn{10}{|c|}{ Características dos participantes } \\
\hline $\bar{x}$ & $-0,44$ & 0,99 & 0,99 & & $-0,76$ & 1,00 & 1,00 & & & \\
\hline \pm & 1,22 & 0,80 & 0,78 & & 1,84 & 0,86 & 0,86 & & & \\
\hline Máx. & 3,66 & 5,94 & 4,51 & & 5,91 & 6,32 & 6,30 & & & \\
\hline Mín. & $-4,10$ & 0,03 & 0,03 & & $-5,14$ & 0,03 & 0,03 & & & \\
\hline
\end{tabular}

$\theta$ nível de habilidades das pessoas (tetha)/nível de dificuldade dos itens; $r=$ correlação de Pearson; $p=$ probabilidade; $\bar{X}=$ média; $\pm=$ desvio padrão; Máx. $=$ máximo; Mín.=mínimo; $D I F=$ Função diferencial do item.

De acordo com o método de ancoragem empregado, observa-se na Tabela 2 que, para as duas versões da escala, a distribuição do nível de dificuldade dos itens encontra-se ao redor da média, cobrindo o intervalo de $0,9 \log$ (entre $-0,54$ e 0,36 ) na versão brasileira e 1,11 $\log$ (entre $-0,42$ e 0,69 ) na versão franco-canadense. Quando comparadas as características dos participantes (Tabela 2) verifica-se que os itens apresentam médias de dificuldade superiores aos níveis de theta dos participantes $(-0,44$, amostra brasileira e $-0,76$, amostra franco-canadense). Além disso, as estatísticas "mínimo" e "máximo" apresentam níveis de theta dos participantes que variam entre $-4,10$ e 3,66 para a amostra brasileira e entre -5,14 e 5,91 para a amostra franco-canadense, indicando que ambas as amostras foram compostas por participantes com diferentes níveis de intensidade no construto.

Em relação às estatísticas descritivas referentes aos índices de ajustes Infit/Outfit apresentados pelos itens de ambas as versões (parte inferior da Tabela 2), verificam-se que estes podem ser classificados como bons, uma vez que se encontram no intervalo entre 0,50 e 1,50. Outro indicador da qualidade dos itens diz respeito aos índices de correlação entre o item e o nível de habilidade dos participantes, iguais ou superiores a 0,72 para a versão brasileira e iguais ou superior a 0,75 para a versão franco-canadense. Em relação aos índices de ajuste referentes aos padrões de resposta dos participantes (Tabela 2), os valores médios infit/ outfit $(0,99 / 0,99$, respectivamente para a amostra brasileira) e (1,00/1,00, respectivamente para a amostra franco-canadense) indicam, no geral, adequação das respostas dos participantes ao padrão esperado pelo modelo. No entanto, as estatísticas mínimo e máximo apontam pessoas que apresentaram padrão inesperado de resposta, haja vista que estes valores extrapolam o intervalo estabelecido como adequados, entre 0,50 e 1,50 .

Por fim, apresentam-se na Tabela 3 as avaliações de DIF em função das diferentes amostras. Na Tabela 3 é possível verificar que, ao analisar os indicadores de DIF (DIF-Contraste $>0,50$ e Probabilidade MantelHaenzel <0,50), os itens 1 ("Meus pensamentos me causam angústia ou sofrimento emocional") e 3 ("Eu analiso tanto as situações, que chega ao ponto em que esta análise passa a não ser mais útil para mim") apresentaram funcionamento diferencial de acordo com a origem da amostra. O item 1 mostrou-se significativamente mais fácil de ser endossado pelos participantes da amostra brasileira $(b=-0,36)$ quando 
TABELA 3

Análise de DIF em função da origem dos participantes nas diferentes amostras

\begin{tabular}{|c|c|c|c|c|c|c|c|c|}
\hline \multirow{2}{*}{ Item } & \multicolumn{4}{|c|}{ Amostra brasileira } & \multicolumn{4}{|c|}{ Amostra franco-canadense } \\
\hline & $\theta 1$ & $\theta 2$ & DIF-Contraste & p Mantel-Haenzel & $\theta 1$ & $\theta 2$ & DIF-Contraste & p Mantel-Haenzel \\
\hline i1 & $-0,07$ & $-0,45$ & 0,38 & 0,00 & 0,49 & 0,40 & 0,09 & 0,48 \\
\hline i2 & 0,31 & 0,45 & $-0,14$ & 0,02 & 1,19 & 0,97 & 0,21 & 0,15 \\
\hline i3 & 0,33 & 0,45 & $-0,12$ & 0,37 & $-0,79$ & $-0,56$ & $-0,23$ & 0,15 \\
\hline i4 & 0,13 & $-0,02$ & 0,16 & 0,03 & 0,13 & 0,17 & $-0,04$ & 0,37 \\
\hline i5 & $-0,12$ & $-0,18$ & 0,06 & 0,39 & $-0,39$ & $-0,36$ & $-0,03$ & 0,37 \\
\hline i6 & $-0,72$ & $-0,36$ & $-0,36$ & 0,00 & $-0,68$ & $-0,55$ & $-0,12$ & 0,58 \\
\hline i7 & 0,17 & 0,14 & 0,03 & 0,80 & 0,11 & $-0,09$ & 0,20 & 0,04 \\
\hline
\end{tabular}

$\theta$ nível de dificuldade dos itens; $p=$ probabilidade; $D I F=$ Função diferencial do item.

comparado à amostra franco-canadense $(b=0,31)$, sendo o oposto encontrado em relação ao item 3, significativamente mais fácil de ser endossado pelos participantes da amostra franco-canadense $(b=-0,39)$ em relação à amostra brasileira $(b=0,43)$. Quando avaliados os indicadores de DIF em função do gênero dos participantes, dentro das amostras de origem, os resultados indicaram que nenhum item apresentou valores de DIF-Contraste iguais ou superiores a 0,50, o que sugere a invariância dos parâmetros dos itens entre tais estratos amostrais avaliados.

\section{Discussão}

Esta pesquisa teve como principal objetivo a estimação de novas evidências de validade e precisão do CFQ para a população brasileira e avaliação da invariância da dificuldade dos itens entre amostras oriundas de diferentes culturas (brasileira e francocanadense), bem como entre participantes da mesma amostra em função do gênero. A partir da AFE verificou-se replicabilidade do modelo unidimensional, originalmente proposto por Gillanders et al. (2014). Este dado também foi observado por outros pesquisadores que avaliaram versões do CFQ adaptadas para outras culturas (Dionne et al., 2016; Romero-Moreno et al., 2014; Ruiz et al., 2017). Especificamente em relação ao estudo desenvolvido por Dionne et al. (2016), os referidos autores utilizaram métodos de extração e retenção de fatores semelhantes ao da presente pesquisa: ULS e AP, respectivamente. Contudo, estes procedimentos foram estimados a partir de matrizes de correlação de Pearson. Neste sentido, os resultados aqui obtidos, tendo como referências as matrizes de correlação policóricas (Lara \& Alexis, 2014) e diferentes métodos de retenção de fatores como Gutman-Kaiser, AP e Método Hull (LorenzoSeva et al., 2011), indicaram a adequação da estrutura proposta ao instrumento, haja vista que esta se manteve inalterada mesmo quando estimada por meio de diferentes métodos. $\mathrm{O}$ mesmo argumento pode, ainda, ser aplicado aos estudos que verificaram adequação da solução unifatorial por meio de AFC como no primeiro estudo da versão brasileira (Lucena-Santos et al., 2017), na versão espanhola (Romero-Moreno et al., 2014) e na versão colombiana (Ruiz et al., 2017).

Assim, a obtenção de novas evidências de validade com base na estrutura interna da versão brasileira do CFQ (AERA, APA, \& NCME, 2014), frente a uma amostra composta por participantes de ambos os gêneros, atende ao primeiro objetivo proposto para este estudo. A estrutura fatorial obtida coincide com a proposta original do instrumento (Gillanders et al., 2014) e corrobora os resultados encontrados internacionalmente (Dell'Orco, 2012; Lucena-Santos et al., 2017; Solé et al., 2015; Wei-chen et al., 2014).

Quanto aos indicadores de precisão dos CFQ obtidos na presente pesquisa, pode-se inferir que este é coerente com os valores observados em diferentes amostras avaliadas com o instrumento original entre 0,88 a 0,92 (Gillanders et al., 2014), por Lucena-Santos et al. (2017) entre 0,92 e 0,94 em mulheres brasileiras, de 0,87 por Romero-Moreno et al. (2014) para versão espanhola, entre 0,89 e 0,93 para versão colombiana (Ruiz et al., 2017) e entre 0,93 e 0,94 para versão franco-canadense (Dionne et al., 2016). Estes valores podem ser interpretados como indicadores desejáveis de precisão (Tabachnick \& Fidel, 2013). Desta forma, pode-se inferir que o segundo objetivo da presente pesquisa, a obtenção de novas evidências de precisão da versão brasileira do $\mathrm{CFQ}$, foi satisfatoriamente alcançado (AERA, APA, \& NCME, 2014).

A partir do índice de correlação de Pearson verificou-se associação negativa moderada entre os escores apresentados pelos participantes nos instrumentos CFQ e ESV, corroborando a hipótese 
teórica, bem como os resultados observados em outros estudos (Gillanders et al., 2014; Romero-Moreno et al., 2014; Ruiz et al., 2017). Este dado sustenta a inferência de evidência de validade divergente entre as variáveis estudadas.

Os resultados verificados por meio da TRI indicaram boa qualidade dos itens, para ambas as versões do CFQ avaliadas separadamente (brasileira e francocanadense). Observou-se índices de ajuste Infit/outfit dentro do intervalo considerado producente. Sugere-se que os mesmos apresentam padrão de resposta esperado pelo modelo quando os níveis de theta das pessoas e os níveis de dificuldade dos itens estão próximos (no caso do Infit), bem como quando os níveis de dificuldade dos itens e theta dos participantes são distantes (Outfit) (Linacre, 2015). Outro indicador de qualidade dos itens observado nesta pesquisa foi o alto índice de correlação entre os itens e o nível de theta dos participantes, o que indica a capacidade dos itens em recuperar a localização dos respondentes no construto.

Contudo, os resultados também apontaram algumas limitações do instrumento, como a restrição na faixa de theta coberta pelos itens de ambas as versões do instrumento, 0,90 log para versão brasileira e 1,11 log para a versão franco-canadense. Tais resultados ficam mais evidentes quando comparados à extensão dos níveis de theta apresentado pelos participantes das diferentes amostras, entre 7,76 logs para a amostra brasileira e entre $11,05 \operatorname{logs}$ para a amostra francocanadense.

Conclui-se que ambas as versões contam com itens adequados para avaliação de sujeitos com níveis de traço latentes próximos da média e, portanto, carecem de itens que possam avaliar, com acurácia, sujeitos que apresentam valores de theta fora deste intervalo. Vale ressaltar que comportamentos semelhantes dos itens são comumente observados em escalas com números reduzidos de itens (Machado, Hauck Filho, Teixeira, \& Bandeira, 2014; Zanini \& Peixoto, 2016). Além disso, o melhor funcionamento de itens para avaliação da extensão central do construto latente parece adequado para avaliação de populações não clínicas, como no caso das amostras avaliadas.

Em relação a análise de DIF foram encontrados dois itens com indicadores de funcionamento diferencial, são eles os itens 1) "Meus pensamentos me causam angústia ou sofrimento emocional" e 3) "Eu analiso tanto as situações, que chega ao ponto em que esta análise passa a não ser mais útil para mim". Enquanto o primeiro recebe pontuações mais altas por parte dos participantes da amostra brasileira, o segundo recebe pontuações mais altas por parte dos participantes da amostra franco-canadense.
Esses resultados denunciam que possíveis diferenças nos escores entre os participantes das diferentes amostras nestes itens podem ser influenciadas por outros vieses, diferentes do construto latente, visto que a comparação entre diferentes grupos é controlada pelo nível no traço latente. Por outro lado, as comparações em função das diferenças culturais seriam inadequadas, pois diferenças entre os grupos poderiam ser resultados de erros atribuídos aos itens dos instrumentos e não a reais diferenças no traço latente (ITC, 2016; Milfont \& Fisher, 2010).

Uma hipótese explicativa para esse dado poderia se dar em função da própria interpretação do item e a forma como as diferentes amostras enfrentam seus problemas. Em um estudo de evidências de validade do Coping Response Inventory (Zanini, Mendonça, Forns, \& Kirchner, 2010) foi descrito o fator 'Análise lógica' desta escala, cujos itens versam sobre pensar em diferentes formas de resolver o problema. $\mathrm{Na}$ população brasileira, estava relacionado a problemas de comportamento, enquanto que, na população americana, estava associado a indicadores de saúde mental e enfrentamento direto.

As autoras discutiram esse fato à luz do componente ruminativo desse fator presente na amostra brasileira e não observado na amostra normativa americana. Mais especificamente, quando os brasileiros pensam em diferentes formas de resolver seus problemas, esse pensamento tende a ser improdutivo à luz do que o item 3 da CFQ apresenta. Por outro lado, na população americana, esse fator estava associado a estratégias de coping mais ativas. Estudos posteriores deveriam avaliar se esse fenômeno pode também estar provocando diferenças nas respostas dos itens entre as amostras brasileiras e franco-canadense, observadas neste estudo.

Não foram observados indicadores de DIF quando avaliados participantes da mesma amostra em função do gênero, o que leva à hipótese de que as diferenças observadas podem estar ligadas apenas a diferenças interculturais. Esta hipótese encontra apoio também nos resultados observados por Ruiz et al. (2017), que observaram invariância dos parâmetros do modelo de medida entre diferentes amostras colombianas: universitários, população geral e pacientes em psicoterapia. Também Lucena-Santos et al. (2017) verificaram invariância do modelo de medida ao comparar diferentes grupos de mulheres brasileiras: população geral, estudantes universitárias e pacientes em tratamento de obesidade e sobrepeso. Em suma, os resultados observados não sustentam evidência de invariância dos parâmetros dos CFQ para estudos de comparação interculturais, embora suportem tais 
evidências para comparação em função do gênero dos participantes em cada amostra.

Apesar de não serem claros os critérios a serem considerados com itens que apresentam DIF (Sass, 2011), diferentes procedimentos têm sido propostos na literatura para diminuir as influências destes itens. Dentre elas, destaca-se a indicação de que estes sejam reformulados (Hambleton, 2005). Para tanto, Damasio et al. (2016) sugerem que, antes da reformulação dos itens, os pesquisadores devem certificar-se da compreensão dos motivos culturais que levaram às diferenças na estimação dos parâmetros, pois somente o conhecimento aprofundado destas características levariam ao desenvolvimento de itens culturalmente equivalentes.

Outros autores ainda sugerem que os itens sejam excluídos e que as comparações entre culturas sejam realizadas com base naqueles que suportem evidências de invariância (Byrne, Shavelson, \& Muthén, 1989). Considerando que o CFQ é um instrumento composto por poucos itens adequados para avaliação de uma faixa restrita dos níveis de theta, a exclusão de dois itens poderia prejudicar a precisão da estimativa do escore latente. Diante desta situação, Damasio et al. (2016) apontam para outro procedimento baseado na TRI, a equalização de itens. Neste caso, os itens com melhores qualidades psicométricas, ou seja, livres de DIF, são usados como âncoras no procedimento de calibração dos diferentes instrumentos em uma escala comum. Isso possibilita a comparação do desempenho dos participantes de diferentes culturas em todos os itens do instrumento, mesmo aqueles que não sustentavam inicialmente evidências de equivalência entre as amostras (von Davier \& von Davier, 2011).

Pode-se concluir que a presente pesquisa possibilitou novas informações quanto ao potencial da versão brasileira do CFQ ao disponibilizar novas evidências de validade e precisão. Os resultados sugerem que o instrumento é uma medida adequada da fusão cognitiva em adultos, confirmando a hipótese teórica sobre a estrutura unidimensional. Destaca-se que o uso da TRI possibilitou levantamento de características das diferentes versões do instrumento, brasileiro e franco-canadense, não verificadas nos estudos anteriores (nível de dificuldade e ajuste dos itens). Além de características dos participantes (theta) e a verificação da invariância dos parâmetros dos itens em função do gênero dos participantes, dentro das respectivas amostras.

Observa-se, contudo, que algumas fragilidades do instrumento foram verificadas, como a limitação na faixa de theta coberta pelos itens das escalas e dois itens que não sustentaram o pressuposto de invariâncias entre as diferentes culturas. Por fim, destacam-se alguns dos limites da presente pesquisa, como contar com amostras por conveniência e acessadas por um único método (online). Em pesquisas futuras, esforços devem ser realizados no sentido de envolver amostras probabilísticas acessadas por outros métodos, como lápis e papel, assim como amostras clínicas, a fim de que a amplitude de nível de habilidade da amostra possa ser ampliada.

\section{Referências}

American Educational Research Association (AERA), American Psychology Association (APA) \& National Council on Measurement in Education (NCME). (2014). Standards for Educational and Psychological Testing. Washington, DC: American Educational Research Association. (DOI INEXISTENTE)

Barbosa, L. M. \& Murta, S. G. (2014). Terapia de aceitação e compromisso: história, fundamentos, modelo e evidências. Revista Brasileira de Terapia Comportamental e Cognitiva, 16(3), 34-49. doi:10.31505/rbtcc.v16i3.711

Byrne B. M., Shavelson R. J., \& Muthén B. (1989). Testing for the equivalence of factor covariance and mean structures: The issue of partial measurement invariance. Psychological Bulletin, 105, 456-466. doi:10.1037/00332909.105.3.456

Costello, A. B., \& Osborne, J. W. (2005). Best Practices in Exploratory Factor Analysis: Four recommendations for getting the most from your analysis. Practical Assessment, Research, and Evaluation, 10(7), 1-9. (DOI INEXISTENTE)

Damásio, B., Valentini, F., Núñes-Rodriguez, S., Kliem, S., Koller, S., Hinz, A., \& Zenger, M. (2016). Is the General SelfEfficacy Scale a Reliable Measure to be used in Cross-Cultural Studies? Results from Brazil, Germany and Colombia. The Spanish Journal of Psychology, 19, E29. doi:10.1017/sjp.2016.30

Dell'Orco, F., Prevedini, A., Oppo, A., Prestes, G. B., \& Moderato, P. (2012). Validation study of the Italian version of the Cognitive Fusion Questionnaire (CFQ). Poster session presented at ACBS Annual World Conference X, Washington D.C., USA. (DOI INEXISTENTE)

Dionne, F., Gagnon, J., Balbinotti, M., Peixoto, E. M., Martel, M-E, Gillanders, D., \& Monestes, J-L. Buying into thoughts: French validation of the Cognitive Fusion Questionnaire. Canadian Journal of Behavioral Science, 48(4), 278-285. doi:10.1037/cbs0000053 
Gillanders, D. T., Bolderston, H., Bond, F. W., Dempster, M., Flaxman, P. E., Campbell, L., ... Remington, R. (2014) The development and initial validation of The Cognitive Fusion Questionnaire. Behavior Therapy, 45, 83-101. doi:10.1016/j.beth.2013.09.001

Gonçalves, D. M. \& Kapczinski, F. (2008). Transtorno mental, indicadores demográficos e satisfação com a vida. Revista de Saúde Pública, 42(6), 1060-1066. doi:10.1590/S0034-89102008000600012

Gouveia, V. V., Chaves, S. S. S., Oliveira, I. C. P., Dias, M. R., Gouveia, R. S. V., \& Andrade, P. R. (2003). A utilização do QSG-12 na população geral: estudo de sua validade de construto. Psicologia: Teoria e Pesquisa, 19(3), 241-248. doi:10.1590/S0102-37722003000300006

Haeys, S. C., Luoma, J. B., Bond, F. W., Masuda, A., \& Lillis, J. (2006). Acceptance and commitment therapy: model, processes and outcomes. Behavior Research and Therapy, 44, 1-25. doi:10.1016/j.brat.2005.06.006

Hayes, S. C., Pistorello, J., \& Biglan, A. (2008). Terapia de aceitação e compromisso: modelo, dados e extensão para a prevenção do suicídio. Revista Brasileira de Terapia Comportamental e Cognitiva, 10, 81-104. doi:10.31505/rbtcc. v10i1.234

Hambleton R. K. (2005). Issues, designs and technical guidelines for adapting tests into multiple languages and cultures. In R. K. Hambleton, P. F. Merenda, \& C. D. Spielberger (Eds.), Adapting educational and psychological tests for cross-cultural assessment (pp. 3-38). Mahwah, NJ: Erlbaum. (DOI INEXISTENTE)

Holgado-Tello, F. P., Chacón-Moscoso, S., Barbero-García, I., \& Vila-Abad, E. (2010). Polychoric versus Pearson correlations in exploratory and confirmatory factor analysis of ordinal variables. Quality \& Quantity, 44, $153-166$. doi:10.1007/s11135-008-9190-y

International Test Commission. (2010). International Test Commission Guidelines for translating and adapting tests. Liverpol: International Test Commission. Recuperado de http://www.intestcom.org

Lara, D. \& Alexis, S. (2014). ¿Matrices policóricas/tetracóricas o matrices Pearson? Un estudio metodológico. Revista Argentina de Ciencias del Comportamiento, 6(1), 39-48. (DOI INEXISTENTE)

Linacre J. M. (2015). A user's guide to Winsteps Ministep: Rasch-model computer programs. Recuperado de http://www. winsteps.com

Lorenzo-Seva, U. \& Ferrando, P. J. (2013). FACTOR 9.2 A Comprehensive Program for Fitting Exploratory and Semiconfirmatory Factor Analysis and IRT Models. Applied Psychological Measurement, 37(6), 497-498. doi:10.1177/0146621613487794

Lorenzo-Seva, U., Timmerman, M. E., \& Kiers, H. A. L. (2011). The hull method for selecting the number of common factors. Multivariate Behavioral Research, 46, 340-364. doi:10.1080/00273171.2011.564527

Lucena-Santos, P., Carvalho, S., Pinto-Gouveia, J., Gillanders, D., \& Oliveira, M. S. (2017). Cognitive Fusion Questionnaire: Exploring measurement invariance across three groups of Brazilian women and the role of cognitive fusion as a mediator in the relationship between rumination and depression. Journal of Contextual Behavioral Science, 6, 53-62. doi:10.1016/j.jcbs.2017.02.004

Machado, W. L., Hauck Filho, N., Teixeira, M. A. P., \& Bandeira, D. R. (2014). Análise de Teoria de Resposta ao Item de Marcadores Reduzidos da Personalidade. Psico, 45(4), 551-558. doi:10.15448/1980-8623.2014.4.13138

Milfont, T. L. \& Fisher, R. (2010). Testing measurement invariance across groups: Applications in cross-cultural research. International Journal of Psychological Research, 3, 111-121. doi:10.21500/20112084.857

Romero-Moreno, R., Marquez-Gonzalez, M., Losada, A., Gillanders, D., \& Fernandez-Fernandez, V. (2014). Cognitive fusion in dementia caregiving: Psychometric properties of the Spanish version of the "Cognitive Fusion Questionnaire". Behavioral Psychology/Psicologia Conductual, 22, 117-132. (DOI INEXISTENTE)

Ruiz, F. J., Suárez-Falcón, J. C., Riaño-Hernandez, D., \& Gillanders, D. (2017). Psychometric Properties of the Cognitive Fusion Questionnaire in Colombia. Revista Latinoamericana de Psicología, 49, 80-87. doi:10.1016/ j.rlp.2016.09.006

Sass, D. A. (2011). Testing measurement invariance and comparing latent factor means within a confirmatory factor analysis framework. Journal of Psychoeducational Assessment, 29(4), 347-363. doi:10.1177/073428291140 6661

Silva, B. (2015). Fusão cognitiva com impulso alimentar: análise das propriedades psicométricas de uma nova medida e o seu impacto no comportamento alimentar. (Dissertação de Mestrado). Recuperado de https://estudogeral.sib.uc.pt/ bitstream/10316/30996/1/Disserta\%C3\%A7\%C3\%A30\%20B\%C3\%A1rbara\%20Leite\%20Silva.pdf

Solé, E., Racine, M., Castarlenas, R., de la Vega, R., Tomé-Pires, C., Jensen, M., \& Miró, J. (2015). The psychometric properties of the cognitive fusión questionnaire in adolescents. European Journal of Psychological Assessment, 32(3), 181-186, doi:10.1027/1015-5759/a000244

Tabachnick, B. G. \& Fidell, L. S. (2012). Using multivariate statistics. New Jersey: Pearson.

Timmerman, M. E. \& Lorenzo-Seva, U. (2011). Dimensionality Assessment of Ordered Polytomous Items with Parallel Analysis. Psychological Methods, 16, 209-220. doi:10.1037/a0023353

von Davier A. A. (2011). A statistical perspective on equating test scores. In A. A. Von Davier (Ed.), Statistical models for test equating, scaling, and linking (pp. 1-17). New York, NY: Springer. doi:10.1007/978-0-387-98138-3

Wei-Chen, Z., Yang, J., Li, X., Hui-Na, G., \& Zhuo-Hong, Z. (2014). Reability and validity of the chinese version of the Cognitive Fusion Questionnaire. Chinese Mental Health Journal, 28, 40-44. (DOI INEXISTENTE)

Zanini, D. S. \& Peixoto, E. M. (2016). Social Support Scale (MOS-SSS): Analysis of the psychometric properties via item response theory. Paidéia, 26(65), 359-368. doi:10.1590/1982-43272665201612 
Zanini, D. S., Mendonça, H., Forns, M., \& Kirchner, T. (2010). Psychometric properties of Coping Response Inventory with brazilian adolescents. Psychological Reports, 107, 617-628. doi:10.2466/08.17.20.PR0.107.5.617-628

Dados dos autores:

Evandro Morais Peixoto - Doutor, Universidade de Pernambuco.

Giovanna Corte Honda - Doutora, Universidade Nove de Julho (UNINOVE).

Joel Gangon - Doutorando, Université du Québec à Trois-Rivieres (UQTR).

Glaucia M. Ataka da Rocha - Doutora, Universidade Federal do Tocantins (UFT).

Tatiana de Cássia Nakano - Doutora, Pontifícia Universidade Católica de Campinas (PUCC).

Daielela Sacramento Zanini - Doutora, Pontifícia Universidade Católica de Goiás (PUCG).

Marcos Alencar Abaide Balbinotti -Doutor, Université du Québec à Trois-Rivieres (UQTR).

Endereço para correspondência:

Evandro Morais Peixoto

Rua Antônio Andrade Lima, 105, apto 101 - São José

55295-345, Garanhuns, Pernambuco, Brasil

E-mail: evandro.peixoto@upe.br

Recebido em: 01/07/2017.

Aceito em: 10/09/2018.

Publicado em: 09/05/2019. 